\title{
Sustained pharmacological inhibition of $\delta P K C$ protects against hypertensive encephalopathy through prevention of blood-brain barrier breakdown in rats
}

\author{
Xin Qi,1 Koichi Inagaki,1 Raymond A. Sobel,1,2 and Daria Mochly-Rosen'1 \\ 1Department of Chemical and Systems Biology and 2Department of Pathology, Stanford University School of Medicine, Stanford, California, USA.
}

\begin{abstract}
Hypertensive encephalopathy is a potentially fatal condition associated with cerebral edema and the breakdown of the blood-brain barrier (BBB). The molecular pathways leading to this condition, however, are unknown. We determined the role of $\delta \mathrm{PKC}$, which is thought to regulate microvascular permeability, in the development of hypertensive encephalopathy using $\delta \mathrm{V} 1-1-$ a selective peptide inhibitor of $\delta$ PKC. As a model of hypertensive encephalopathy, Dahl salt-sensitive rats were fed an $8 \%$ high-salt diet from 6 weeks of age and then were infused s.c. with saline, control TAT peptide, or $\delta V 1-1$ using osmotic minipumps. The mortality rate and the behavioral symptoms of hypertensive encephalopathy decreased significantly in the $\delta \mathrm{V} 1-1$-treated group relative to the control-treated group, and $\mathrm{BBB}$ permeability was reduced by more than $60 \%$. Treatment with $\delta \mathrm{V} 1-1$ was also associated with decreased $\delta P K C$ accumulation in capillary endothelial cells and in the endfeet of capillary astrocytes, which suggests decreased microvasculature disruption. Treatment with $\delta \mathrm{V} 1-1$ prevented hypertension-induced tight junction disruption associated with BBB breakdown, which suggests that $\delta$ PKC may specifically act to dysregulate tight junction components. Together, these results suggest that $\delta P K C$ plays a role in the development of hypertension-induced encephalopathy and may be a therapeutic target for the prevention of $\mathrm{BBB}$ disruption.
\end{abstract}

\section{Introduction}

Hypertensive encephalopathy is a complex condition that is characterized by a rapid onset of headache, nausea, impaired consciousness, and visual disturbances (1-3). Hypertensive encephalopathy in humans is associated with breakdown of the blood-brain barrier (BBB) to fluid, cells, and macromolecules, especially in the cerebral cortex and in the basal ganglia (1). The change in permeability mainly affects intracerebral arteries and leads to cerebral edema, which is invariably fatal in the absence of antihypertensive treatment (2). Although antihypertensive treatment reduces the structural changes in the cerebral vessels (4), the molecular basis for hypertensive encephalopathy is not well understood.

The BBB is an essential structure for maintaining CNS homeostasis. It consists of vascular endothelia surrounded by a layer of astrocytic foot processes and microglia (5-7). Tight junctions of the BBB restrict paracellular permeability (8-10). When the barrier integrity is disrupted, inflammatory cells and fluid penetrate the brain, which results in edema and cell death (5-7). Changes in BBB function are documented in several diseases, including stroke, muscular dystrophy, multiple sclerosis, Alzheimer disease, and septic encephalopathy (11-15). Consequently, protection of the BBB has become an important target for drug development.

Nonstandard abbreviations used: AQP4, aquaporine 4; BBB, blood-brain barrier; df, degrees of freedom; DS, Dahl salt-sensitive; ZO-1, zonula occludens 1.

Conflict of interest: Daria Mochly-Rosen is the founder of KAI Pharmaceuticals Inc., a company that plans to bring PKC regulators to the clinic. However, none of the work described here is in collaboration with or supported by the company. The authors have declared that no conflict of interest exists.

Citation for this article: J. Clin. Invest. 118:173-182 (2008). doi:10.1172/JCI32636.
The PKC family of serine/threonine kinases consists of 10 different isozymes, which are further classified into 3 major categories: conventional PKCs $(\alpha, \beta \mathrm{I}, \beta \mathrm{II}$, and $\gamma)$, novel PKCs $(\delta, \varepsilon, \eta$, and $\theta)$, and atypical PKCs $(\zeta, \lambda / \mathrm{I}$, and $\mu)$. PKC $\alpha, \beta \mathrm{I}, \beta \mathrm{II}, \gamma, \delta$, and $\varepsilon$ mRNA and proteins are present in the CNS, which indicates unique cellular and subcellular localizations (16). Direct activation of PKC by phorbol esters increases the flux of fluid and macromolecules through the microvascular wall (17). Inhibitors of PKC, such as H7, calphostin $\mathrm{C}$, and chelerythrine, reduce the increased endothelial permeability that is induced by hydrogen peroxide, neutrophils, and platelet-activating factor (18-20). More importantly, PKC activation and subsequent vascular barrier dysfunction may be involved in the progress of circulatory disorders associated with atherosclerosis (21), ischemia/reperfusion injury (22), and diabetic retinopathy (23). Thus, PKC is considered to be a potential mediator of microvascular permeability under various stimulated conditions. However, specific PKC isozymes have distinct effects on the function and the integrity of epithelial cell and endothelial cell barriers in vitro and in vivo. Thus, direct targeting of specific PKC isozymes may help to identify which PKC isozyme regulates microvascular permeability. Our previous report showed that $\delta \mathrm{PKC}$ plays a deleterious role in stroke and neuronal cell death and that inhibition of $\delta \mathrm{PKC}$ by single injection of the $\delta P K C$ selective inhibitor $\delta V 1-1(0.2 \mathrm{mg} / \mathrm{kg})$ reduces cerebral damage following a middle cerebral artery occlusion stroke model by more than $70 \%$ (24). Furthermore, $\delta$ PKC-null mice exhibit reduced infarction following middle cerebral artery occlusion (25). However, the role of $\delta \mathrm{PKC}$ in hypertension-induced encephalopathy and BBB disruption has not been determined.

A lethal form of hypertension has been shown to develop in Dahl salt-sensitive (DS) rats fed a high-salt diet from an early age (26). 

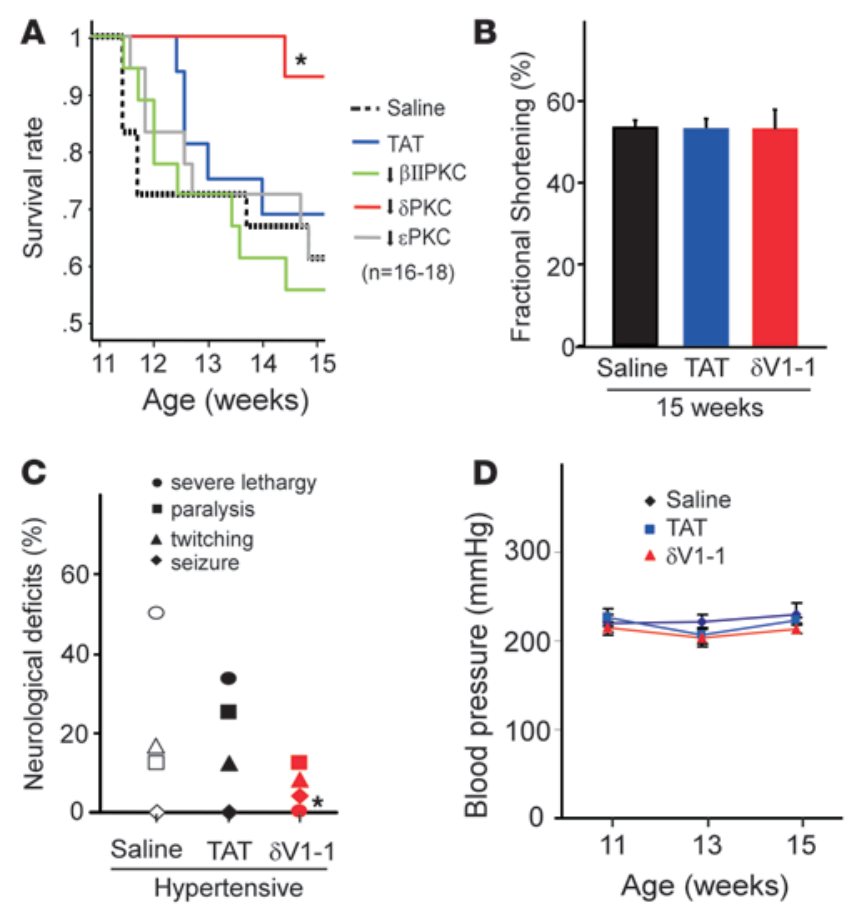

Behavioral symptoms of encephalopathy and stroke, disruption of the $\mathrm{BBB}$, and the occurrence of intracerebral hemorrhage in DS rats fed a high-salt diet were noted $(27,28)$. Using the DS rat model, we investigated the role of $\delta \mathrm{PKC}$ in hypertensive encephalopathy. We used various PKC-selective regulators (29) and found that the $\delta$ PKC-specific peptide inhibitor $\delta V 1-1$ reduced the incidence of hypertension-induced encephalopathy by protecting the integrity of the BBB. The molecular basis of these events was also studied.

\section{Results}

$\delta V 1-1$ decreases the mortality rate of hypertensive rats. Hypertensive DS rats develop symptoms of encephalopathy and stroke between 11 and 15 weeks of age while maintaining normal cardiac functions (30). During this period, about $60 \%$ of the animals die of encephalopathy. Around 16-17 weeks of age, the surviving rats (about $40 \%$ of the animals) begin to develop heart failure, and the animals die at about 21 weeks of age (30). Because we focused on encephalopathy, we only studied animals between the ages of 11 and 15 weeks. The animals were treated with various isozyme-selective PKC inhibitors using an osmotic pump to obtain sustained delivery. A great delay in mortality was observed only in the hypertensive rats that were treated with $\delta \mathrm{V} 1-1$, the $\delta \mathrm{PKC}$-selective inhibitor (Figure 1A). Whereas rats in the vehicle-treated group died by 15 weeks, only 3 of 18 rats in the $\delta V 1-1$-treated group died. Treatment with other PKC isozyme-specific regulating peptides, including the $\varepsilon \mathrm{PKC}$-selective inhibitor and the $\beta$ IIPKC-selective inhibitor, did not significantly affect survival of the hypertensive DS rats. Furthermore, rats treated with $\delta V 1-1$ at the age of 15 weeks showed no improvement in cardiac function (as evaluated by fractional shortening) compared with rats treated with saline or TAT control peptide (Figure 1B). Therefore, it is unlikely that $\delta$ V1- 1 treatment improved survival by ameliorating cardiac dysfunction. Together, these data suggest that, of all the PKC isozymes, $\mathrm{P}$ PKC has a specific deleterious effect, i.e., it induces brain injury in hypertensive rats.

\section{Figure 1}

Sustained treatment with $\delta \mathrm{V} 1-1$ reduces hypertension-induced encephalopathy in the DS rat model. (A) DS rats were fed a high-salt diet $(8 \%$ $\mathrm{NaCl}$ ) from 6 to 15 weeks of age and treated with saline, TAT, or PKC peptide regulators beginning at 11 weeks via s.c. implanted osmotic pumps that delivered peptides at a rate of $1.0 \mathrm{mg} / \mathrm{kg} / \mathrm{d}$. Any seizures, twitchings, paralysis, or lethargy exhibited by the animals was recorded by an observer blinded to the treatments. DS rats were treated with peptide regulators, including inhibitors selective for $\beta I$, $\delta$, or $\varepsilon$ PKC from 11 to 15 weeks. Sustained treatment with the $\delta P K C$ inhibitor $\delta V 1-1$ significantly increased the survival of DS rats $\left({ }^{*} P<0.01\right.$, log-rank test). (B) None of the treatments affected cardiac function measured by fractional shortening at the age of 15 weeks. Data are mean \pm SEM ( $n=12-16$ rats per group). (C) $\delta \mathrm{V} 1-1$ treatment significantly improved the neurological status of DS rats. Individual neurological symptoms were recorded and the percentage of each major behavioral symptom of encephalopathy in each group of animals was monitored $(n=24$ rats per group). ${ }^{*} P<0.01$ versus saline- or TAT-treated group, Fisher's exact test. (D) None of the treatments affected the elevated blood pressure. Data are mean $\pm \operatorname{SEM}(n=24$ rats per group).

$\delta V 1-1$ treatment reduces encephalopathy symptoms in hypertensive rats. We next identified the type and severity of the encephalopathy symptoms using a new group of animals. Because the assessment of symptoms is subjective, the observer was blinded to the treatments. Of the 24 saline-treated rats, 4 exhibited twitching, 3 displayed symptoms of fore- and hind-limb paralysis, and 12 exhibited severe lethargy (the most serious neurological deficit). The TAT-treated group exhibited neurological deficits similar to those of the salinetreated group. In contrast, of the $24 \delta \mathrm{V} 1-1$-treated rats, 0 showed severe lethargy, 1 had a seizure, 2 exhibited twitching behaviors, and 3 had limb paralysis. Rats in the vehicle-treated group with symptoms of encephalopathy usually died within 3 days. However, in the $\delta$ V1-1-treated group, disease was less severe; the 1 rat that had a seizure and the 2 rats that exhibited twitching behaviors did not die of hypertensive encephalopathy. These data suggest that $\delta$ V1-1 may reverse neurological dysfunction. However, because treatment began before the onset of neurological dysfunction, the therapeutic effect of $\delta$ V1-1 after the symptoms occurred could not be discerned. We are currently investigating the benefit of $\delta \mathrm{PKC}$ inhibition treatment on hypertensive encephalopathy once symptoms have manifested. This experiment will help determine whether $\delta$ PKC inhibition will benefit patients with hypertension crisis and encephalopathy symptoms. Here we observed amelioration of the original neurological deficits in the group of rats administered $\delta V 1-1$, which was not observed in the control-treated groups (Figure 1C).

$\delta V 1-1$ has no effect on blood pressure. Because encephalopathy is induced by hypertension, we confirmed that none of the PKC regulators affected blood pressure. In rats, a systolic blood pressure of $150 \mathrm{mmHg}$ is considered the threshold for hypertension (27). DS rats fed $8 \% \mathrm{NaCl}$ for 5 weeks (from 6 to 11 weeks of age) showed severe hypertension $(241 \pm 37 \mathrm{mmHg})$. Sustained treatment with $\delta V 1-1$ from 11 to 15 weeks $(1.0 \mathrm{mg} / \mathrm{kg} / \mathrm{d})$ did not alter the elevated blood pressure as compared with the saline- or TATtreated animals (Figure 1D). 
A
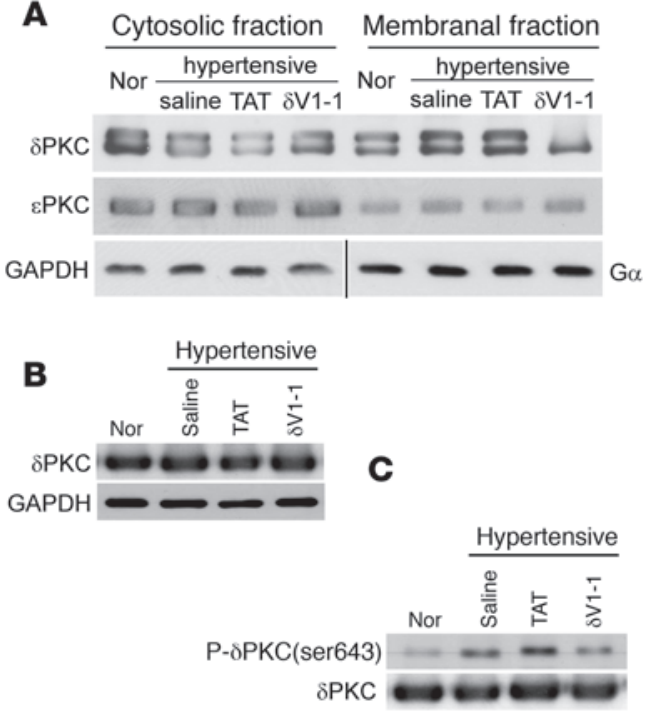
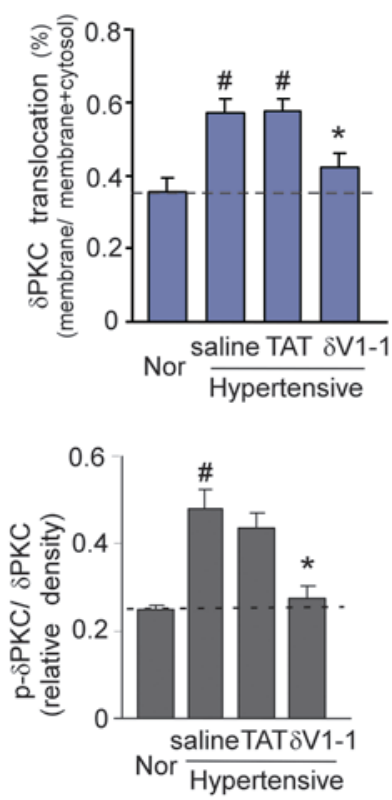

\section{Figure 2}

Sustained delivery of $\delta \mathrm{V} 1-1$ peptide inhibits hypertension-induced $\delta P K C$ translocation. Rats fed a high-salt diet were treated with $\delta \mathrm{V} 1-1$, saline, or TAT as in Figure 1. Brain tissue was harvested at 13 weeks of age. (A) Soluble and particulate fractions of brain tissue were subjected to Western blot analysis, and $\delta$ PKC translocation was determined. The blots show representative results, and the graph provides quantification of $\delta P K C$ translocation from the cytosolic to the membranal fractions. Data are mean \pm SEM $(n=3$ rats per group). $\mathrm{F}=13.68, \mathrm{df}=3 .{ }^{*} P<0.05$ versus saline or TAT treatment; ${ }^{\#} P<0.05$ versus rats fed a low-salt diet (Nor). GAPDH and G $\alpha$ were used as internal controls for cytosolic and membranal fractions, respectively. (B) Total lysates of the brain were subjected to Western blot analysis with anti-SPKC. GAPDH was used as an internal loading control. Representative data are from 3 rats per group. (C) Phosphorylation of $\delta$ PKC (Ser643) was determined by Western blot analysis. Data are mean \pm SEM ( $n=3$ rats per group). $\mathrm{F}=11.49, \mathrm{df}=3 .{ }^{*} P<0.05$ versus saline treatment groups; ${ }^{\#} P<0.05$ versus rats fed a low-salt diet.
Sustained delivery of $\delta V 1-1$ peptide inhibits $\delta P K C$ translocation. We next confirmed that $\delta \mathrm{V} 1-1$, the $\delta \mathrm{PKC}$-selective inhibitor peptide, exerted its biological effect and selectively inhibited $\delta$ PKC activity. Translocation of PKC from the soluble to the particulate fraction is a marker for PKC activation (31). We therefore assessed $\delta \mathrm{PKC}$ translocation in the hypertensive rat model and the effects of the treatments on the translocation. In 13 -week-old rats, there was a $40 \%$ increase in $\delta \mathrm{PKC}$ translocation in the saline- and TAT-treated groups relative to the normotensive rats (Figure $2 \mathrm{~A}$ ), which indicated that $\delta \mathrm{PKC}$ is activated by hypertension. In rats treated with $\delta \mathrm{V} 1-1$, the increase in $\delta$ PKC translocation was reduced by $70 \%$ to only about $15 \%$ above the basal value (Figure 2A). As expected, $\delta V 1-1$ did not affect $\varepsilon$ PKC translocation, which indicated the selectivity of the inhibitor (Figure 2A). Furthermore, there were no changes in the total level of $\delta \mathrm{PKC}$ in any of the treatment groups (Figure $2 \mathrm{~B}$ ). In addition, hypertension-induced phosphorylation of
¿PKC (Ser643) was significantly inhibited by $\delta \mathrm{V} 1-1$ treatment, by $80 \%$, compared with the saline-treated group (Figure $2 \mathrm{C}$ ).

$\delta V 1-1$ reduces $B B B$ permeability. To determine the extent of $\mathrm{BBB}$ disruption caused by hypertension and the effects of $\delta P K C$ inhibition, we evaluated the extravasation of Evans blue dye into the brain parenchyma, a sign of BBB permeability (32). In the TATtreated group, 5 of 6 rats showed Evans blue extravasation into brain parenchyma. In contrast, only 2 of 6 rats treated with $\delta V 1-1$ showed some increase in BBB permeability (Figure 3A). The amount of Evans blue dye in the brain parenchyma was greater in the TAT-treated group $(3.6 \pm 0.7 \mathrm{mg} /$ brain $)$ than in the $\delta \mathrm{V} 1-1-$ treated group $(1.6 \pm 0.3 \mu \mathrm{g} / \mathrm{brain})$ (Figure $3 \mathrm{~B} ; P<0.01)$. As expected, no Evans blue extravasation was observed in control normotensive rats fed a low-salt diet $(0.3 \% \mathrm{NaCl})$.

Immunoreactivity of $\delta P K C$ in brain microvessels in DS rats. Endothelial cell and astrocyte endfeet surrounding the brain capillaries form the $\mathrm{BBB}$ to limit transcellular flux across the vasculature (33). We
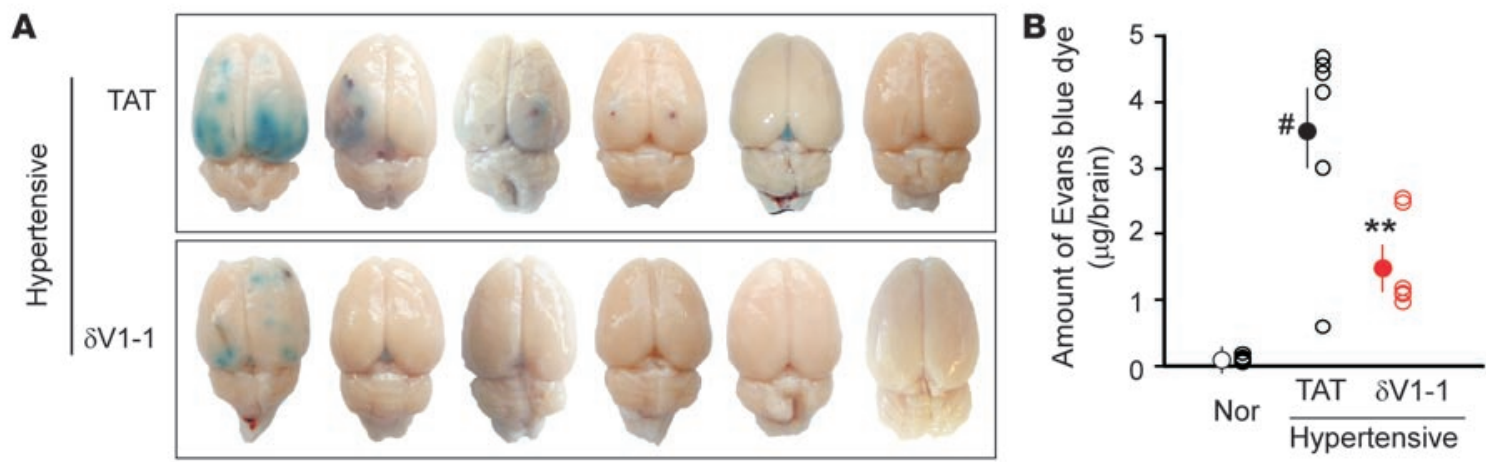

\section{Figure 3}

Sustained $\delta$ V1-1 treatment decreases hypertension-induced increases in the permeability of the BBB. Evans blue dye (30 mg/kg) was injected i.v. at 13 weeks of age in rats fed the high-salt diet. The brains were isolated $30 \mathrm{~min}$ after injection. (A) Evans blue dye extravasation in DS rats treated with TAT or $\delta \mathrm{V} 1-1$. (B) Quantification of Evans blue dye extravasation in the parenchyma of DS rat brain. Open circles represent the amount of Evans blue dye in individual brains, and the filled circles represent the mean. Data are mean \pm SEM $(n=6$ rats per group). F $=17.71$, df $=2 .{ }^{*} P<0.01$ versus TAT treatment groups, ${ }^{*} P<0.05$ versus rats fed the low-salt diet. 
A

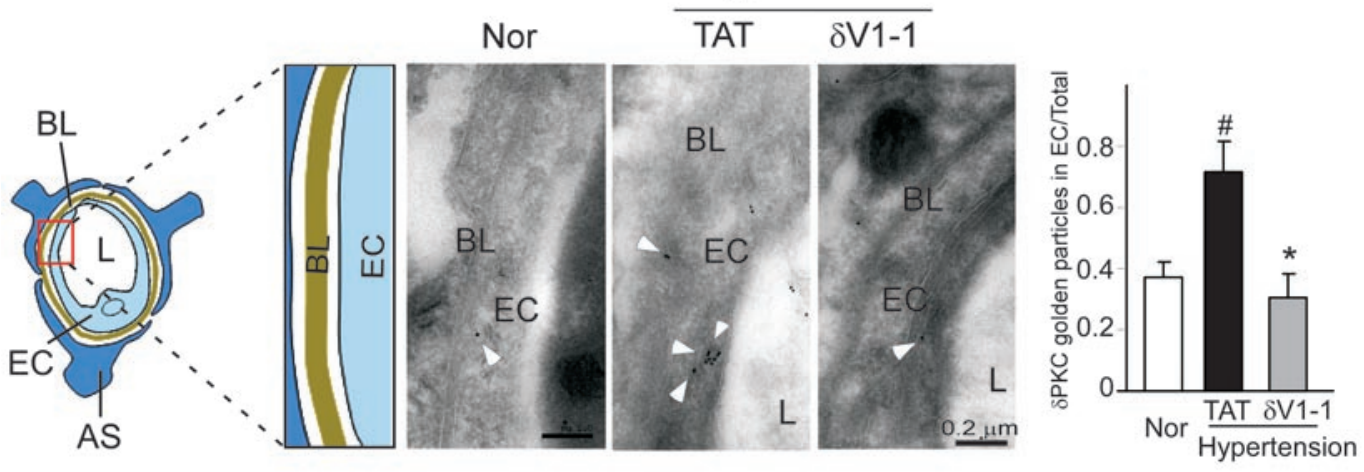

B
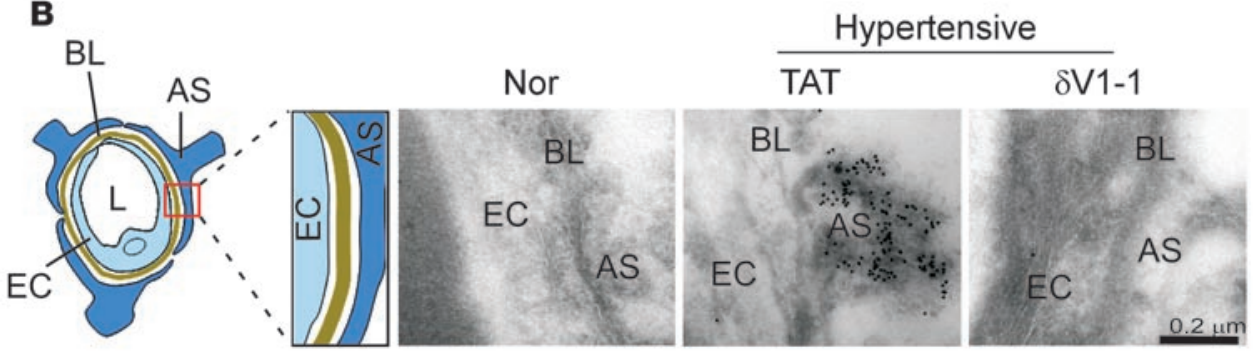

C

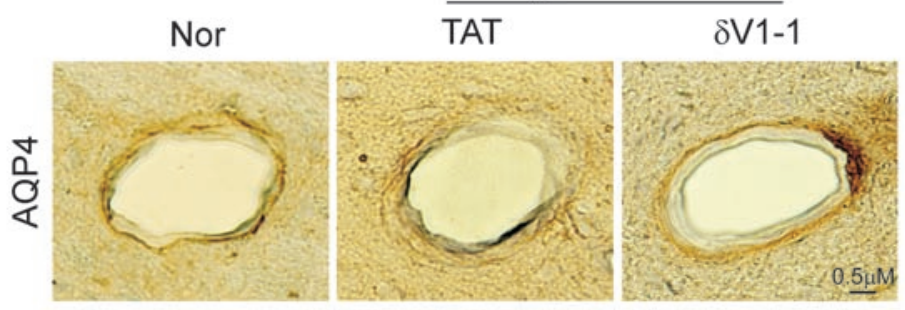

Figure 4

Sustained $\delta \mathrm{V} 1-1$ treatment preserves the integrity of the cerebral capillaries of hypertensive rats. Brain cortex samples were collected from 13-week-old DS rats treated with either TAT or $\delta V 1-1$ and stained with $\delta P K C$ using the immunogold labeling method. (A) Middle: $\delta P K C$ immunogold labeling in endothelial cells and improved morphology of basal lamina (BL) after $\delta \mathrm{V} 1-1$ treatment were noted. $L$, vessel lumen. A scheme of the area studied is shown at left; a histogram depicting quantitative data of gold particles in endothelial cells is shown at right. Data are mean \pm SEM $\left(n=3\right.$ animals per group). $\mathrm{F}=16.75, \mathrm{df}=2 .{ }^{\star} P<0.05$ versus TAT treatment; ${ }^{*} P<0.05$ versus normotensive rats. (B) Increased $\delta P K C$ levels in the endfeet of astrocytes (AS) that envelop vessel walls were seen in rat brains from hypertensive rats, which were blocked by $\delta \mathrm{V} 1-1$ treatment. (C) Immunohistochemical analysis of astrocytes surrounding cerebral vessels. Frozen sections of rats treated with TAT or $\delta \mathrm{V} 1-1$ were stained with AQP4, a marker of astrocyte endfeet process. Immunostaining of AQP4 around the cerebral vessel wall was observed. $n=3$ rats per group.

therefore assessed $\delta \mathrm{PKC}$ localization in these structures using immunogold electron microscopy. In contrast with normotensive DS rats fed a low-salt diet, the TAT-treated hypertensive rats had a discontinuous basal lamina and much thicker capillary endothelial layer in the microvessels and more immunolabeling of $\delta \mathrm{PKC}$ in the endothelial cells forming the border of the capillary (Figure 4A). In contrast, relative to the TAT-treated hypertensive rats, the $\delta \mathrm{V} 1-1-$ treated rats showed a clear basal lamina layer, thin endothelial cell layer, and less $\delta$ PKC labeling in the endothelial cells (Figure 4A, right vs. middle).

Astrocyte swelling indicates $\mathrm{BBB}$ damage. In the hypertensive rats treated with TAT, we found a large number of gold particle (SPKC immunoreactivity) label that was localized in the swollen astrocyte endfeet covering the vessel wall, whereas no gold particles were observed in the normotensive rats (Figure 4B, middle vs. left). Treat- ment with $\delta V 1-1$ reduced $\delta$ PKC labeling, and the shape of astrocyte endfeet appeared normal (Figure 4B, right). The levels of aquaporine 4 (AQP4; a marker of astrocyte endfeet processes) appear reduced when the BBB is disrupted $(34,35)$. Immunohistochemistry assessment showed that the perivascular astrocyte processes enveloping the control vessel walls had a strong AQP4 immunoreactivity in normotensive rats. In the TAT-treated hypertensive rats, the vessels had a more discontinuous and faint staining of AQP4 at their abluminal side, and this abnormal staining was corrected in the $\delta V 1-1$-treated group (Figure 4C). In addition, long-term treatment with $\delta V 1-1$ may alter the density of specific cells, such as cerebral endothelial cells, astrocytes, and pericytes, as a means to improve BBB function. Future studies will address this possibility. Collectively, these data suggest that $\delta$ PKC activation mediates, at least in part, the disruption of brain microvasculature in hypertensive rats. 
A Cytosolic Fraction

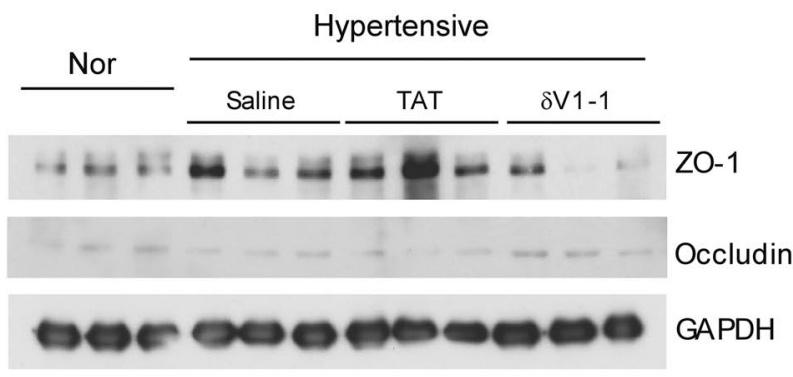

Membrane Fraction (Triton-soluble)

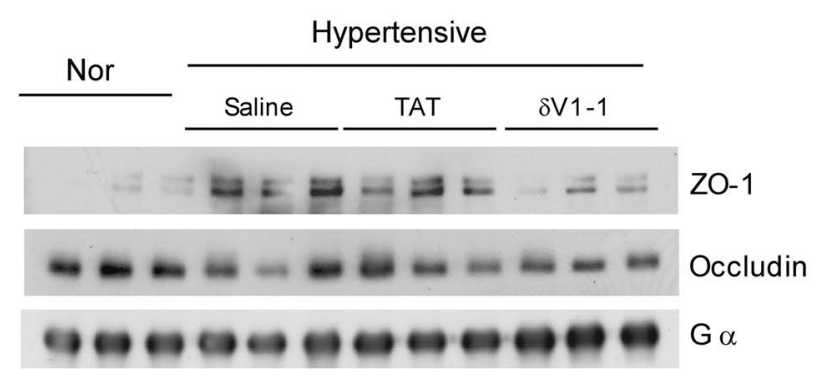

Cytoskeletal Fraction (Triton-insoluble)

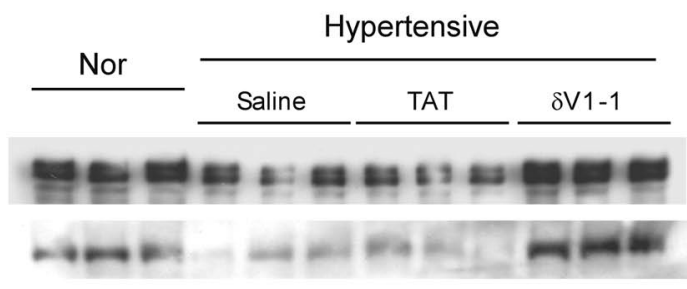

$\mathrm{ZO}-1$

Occludin
B
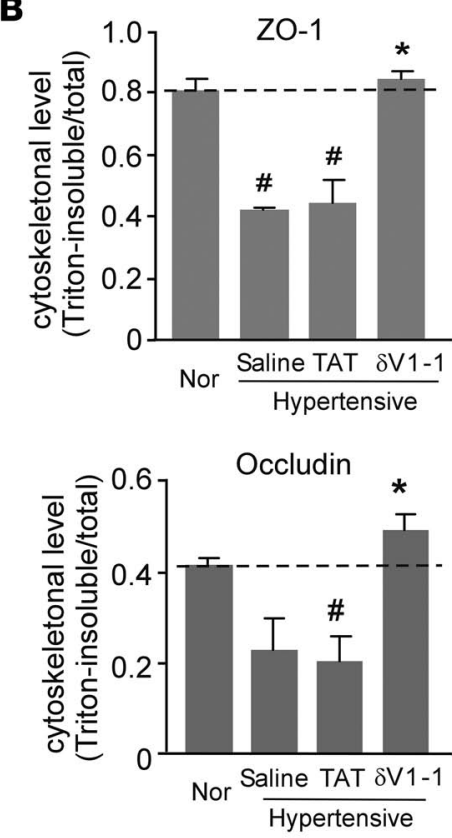

C

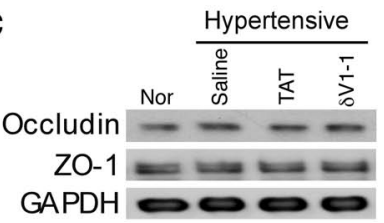

$\delta V 1-1$ blocks disorganization of tight junction in bypertensive DS rats. The tight junction is an essential component of the $\mathrm{BBB}$. A large number of studies showed that altered BBB permeability is associated with disruption of tight junction components $(8-10,13)$. At the molecular level, the tight junction proteins, including claudins, occludin (a transmembrane protein), and $\mathrm{ZO}-1, \mathrm{ZO}-2$, and ZO-3 (members of the membrane-associated guanylate kinase homolog family), interact with each other and with the actin cytoskeleton to form tight junction complexes $(8,9)$. Alteration in occludin and ZO-1 subcellular distribution has been used as a marker for disrupted tight junction and epithelial barrier $(13,35,36)$. We therefore determined the subcellular distribution of ZO-1 and occludin using Western blot analysis of detergent-soluble (membrane and cytosolic fractions) and detergent-insoluble fractions (cytoskeleton-enriched fraction). We found less of the tight junction proteins ( $\mathrm{ZO}-1$ and occludin) in the detergent-insoluble fraction and more in the detergent-soluble fraction (Figure 5A). As compared with normotensive rats, there was a $40 \% \pm 2 \%$ decrease in the level of cytoskeleton-element associated $\mathrm{ZO}-1$ and a corresponding increase in soluble $\mathrm{ZO}-1$ in 13 -week-old hypertensive rats treated with TAT. Treatment with $\delta$ V1-1 significantly blocked this translocation of ZO-1 from the cytoskeleton to the detergent-soluble fraction (Figure 5). We also found that the translocation of occludin from the cytoskeletonelement-associated fraction to the soluble fraction was similar to that of $\mathrm{ZO}-1$ and that $\delta \mathrm{V} 1-1$ treatment blocked this translocation

\section{Figure 5}

Sustained $\delta \mathrm{V} 1-1$ peptide treatment blocks the translocation of ZO-1 and occludin out of the tight junction-enriched cytoskeletal fractions in brains of hypertensive rats. Cytosolic and membranal (detergent-soluble) fractions and cytoskeletal (detergent-insoluble) fraction were isolated from rat brains treated as above. (A) Lysates from DS rat brains were subject to Western blot and analyzed using anti-ZO-1 and anti-occludin antibodies. GAPDH (a cytosolic marker) and $\mathrm{G} \alpha$ (a membranal marker) were used as internal controls. (B) Histogram demonstrating translocation of $\mathrm{ZO}-1$ and occludin out of the cytoskeletonal fractions in TAT-treated rats and its reversal in the $\delta$ V1-1-treated group. Data are mean \pm SEM $(n=3$ rats per group). $\mathrm{F}=27.97, \mathrm{df}=3(\mathrm{ZO}-1)$; $\mathrm{F}=8.84$, df $=3$ (occludin). $\# P<0.05$ versus rats fed lowsalt diet; ${ }^{*} P<0.05$ versus TAT or saline treatment. (C) Total lysates from DS rat brains were subjected to Western blot analysis with anti-ZO-1 and antioccludin antibodies. GAPDH was used as an internal loading control. $n=3$ rats per group.

compared with that in the TAT-treated group (Figure 5). No significant changes in the total protein levels of ZO-1 and occludin were observed in either treatment group (Figure 5C). These data strongly indicate that $\delta \mathrm{V} 1-1$ treatment blocks the disruption of the structure of tight junctions caused by hypertension in the DS rat model.

Next, using immunofluorescence we examined the cellular distribution of these 2 tight junction proteins in the hypertensive DS rat brains treated with TAT or $\delta \mathrm{V} 1-1$. In the normotensive rats, ZO-1 and occludin colocalized with CD31 (an endothelial cell marker) and exhibited discrete dots or line-like staining. However, the staining pattern was altered in hypertensive rat brains treated with TAT; the staining of both tight junction proteins was diffuse and appeared aggregated. This extensive disorganization of ZO-1 and occludin in TAT-treated rats was reversed in the hypertensive rats treated with $\delta V 1-1$ (Figure $6, \mathrm{~A}$ and $\mathrm{B}$ ). Thus, the changes in the cellular distribution of the tight junction proteins $\mathrm{ZO}-1$ and occludin in the hypertensive rats were associated with $\delta$ PKC activation, which suggests that $\delta \mathrm{PKC}$ mediates disruption of the organization of tight junction proteins and the consequent increased vascular permeability and results in BBB breakdown.

$\delta P K C$ phosphorylates ZO-1 in bypertensive rats. ZO- 1 is a phosphorylated protein and contains 34 repeats of a PKC phosphorylation consensus motif, which suggests that ZO-1 is a substrate of PKC signaling (37). Furthermore, ZO-1 phosphorylation correlates with tight junction disruption (38-40). To begin elucidating how $\delta \mathrm{PKC}$ disrupts 
A
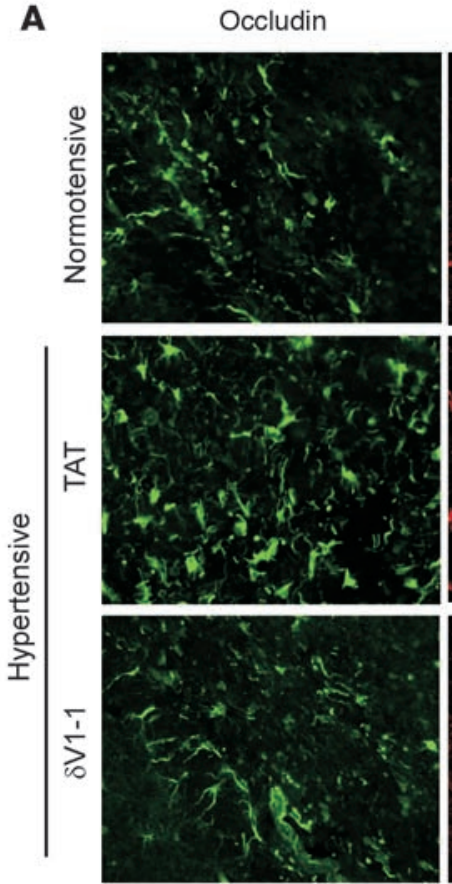

B
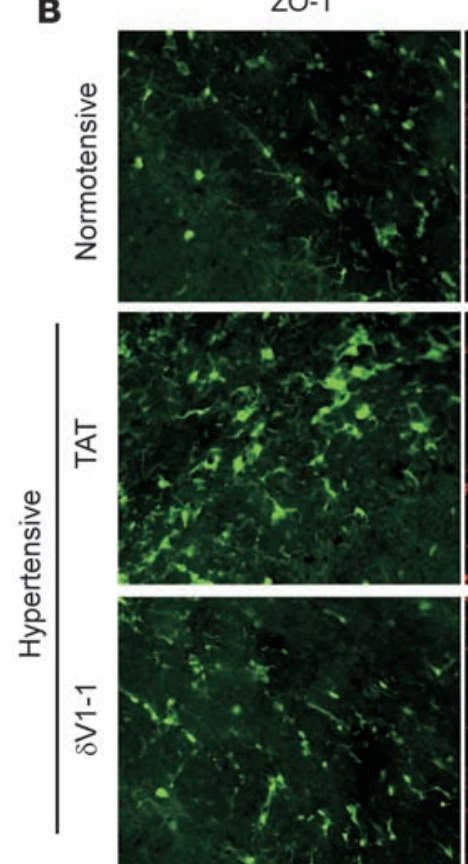

CD31
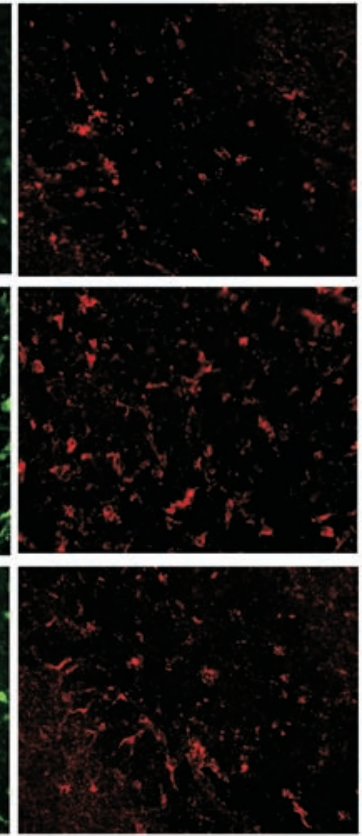

CD31
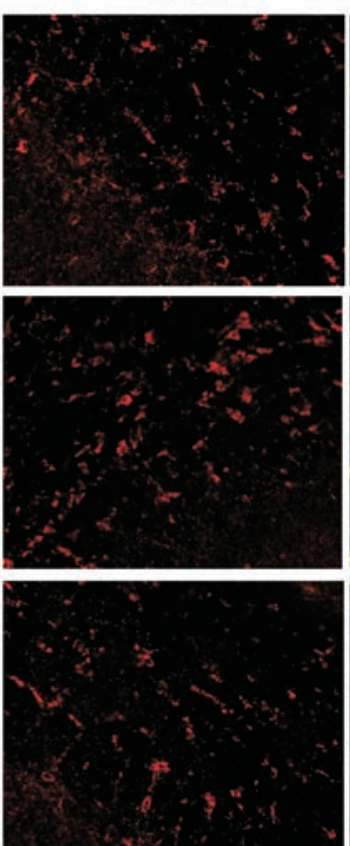

Merge
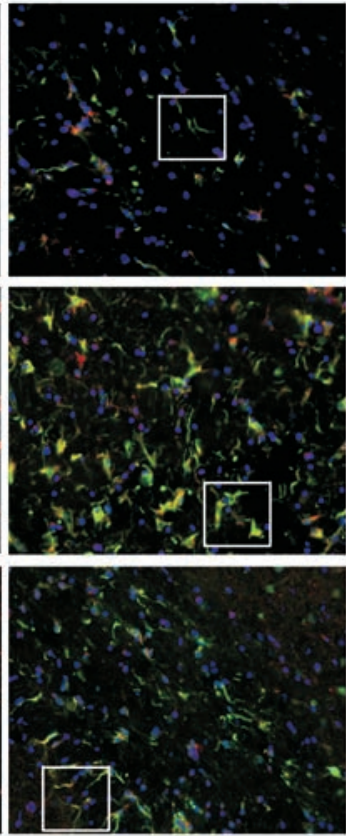

Merge
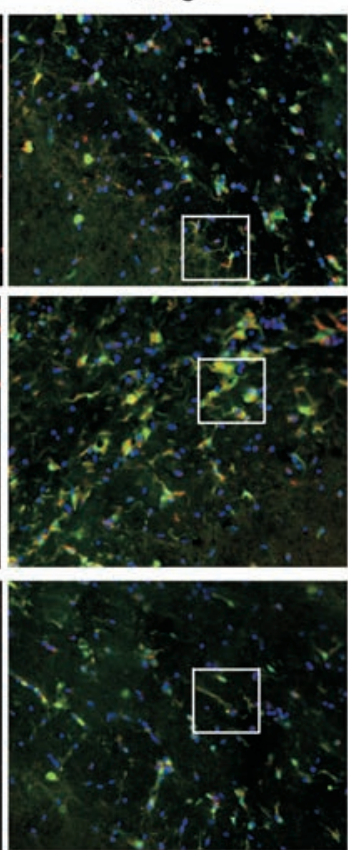

Enlarged area
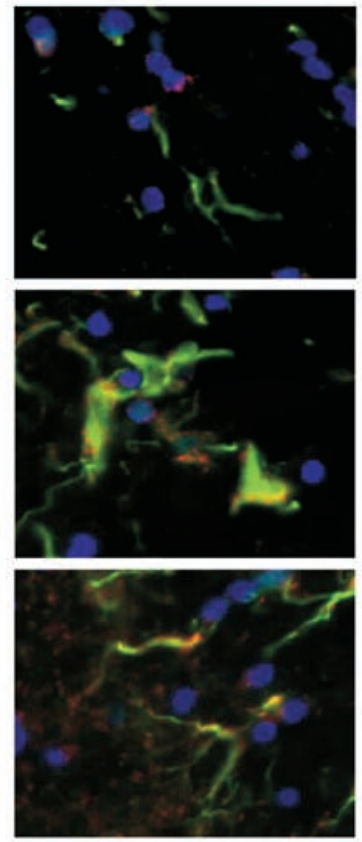

Enlarged area
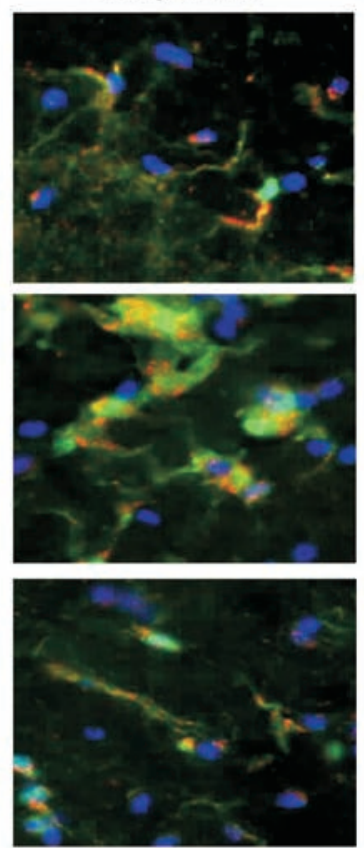

Figure 6

Sustained $\delta \mathrm{V} 1-1$ treatment preserves the cellular distribution of ZO-1 and occludin in hypertensive rat brain. Fresh frozen brain sections (13 weeks of age) were stained with antibodies for occludin (A) or ZO-1 (B) (green). Endothelial cells were identified by CD31 staining (red). Nuclei were stained by Hoechst (blue). Left: typical examples of 3 independent experiments. Scale bar: $0.5 \mu \mathrm{m}$. Right: enlargement of the area marked by the white boxes in the merged image.

tight junctions, we determined the changes in phosphorylation of ZO-1 in the hypertensive DS rat model. In hypertensive DS rats, membrane-associated $\mathrm{ZO}-1$ was phosphorylated twice as much as in the normotensive rats (Figure 7). Importantly, $\delta \mathrm{V} 1-1$ treatment completely blocked this rise in ZO-1 phosphorylation (Figure 7). The data suggest that $\delta \mathrm{PKC}$ phosphorylates $\mathrm{ZO}-1$ directly or increases its phosphorylation indirectly and consequently leads to disruption in ZO-1 function and an increase in BBB permeability.

\section{Discussion}

In the present study, we demonstrated for the first time to our knowledge that sustained inhibition of $\delta \mathrm{PKC}$, using a $\delta \mathrm{PKC}-$ 


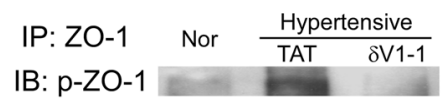

IB: ZO-1

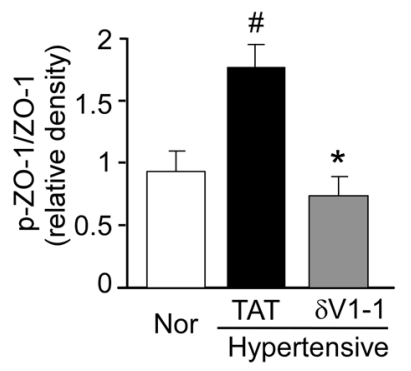

selective inhibitor peptide, significantly reduced the incidence and symptoms of hypertensive encephalopathy in a hypertensive DS rat model. We also found that neuroprotection by the $\delta \mathrm{PKC}$ inhibitor was associated with the prevention of BBB breakdown, although blood pressure remained high.

In DS rats fed a high-salt diet, BBB permeability develops before the appearance of symptoms of brain damage $(27,28)$. We found that $\delta$ V1-1 decreased BBB permeability by about $60 \%$ as measured by Evans blue extravasation. Moreover, inhibition of $\delta \mathrm{PKC}$ in endothelial cells and capillary barrier-associated astrocyte endfeet in the DS rat brain was linked to ameliorating morphological dysfunction in the brain microvasculature. Thus, prevention of BBB permeability via treatment with $\delta V 1-1$

\section{Figure 7}

Sustained treatment with $\delta \mathrm{V} 1-1$ inhibits the serine/threonine phosphorylation of ZO-1 in hypertensive rat brain. Brain tissues were fractionated, and samples from the Triton-soluble membranal fraction were immunoprecipitated with anti-ZO-1 antibody. Top: typical examples of 6 independent experiments. Bottom: quantification of ZO-1 phosphorylation. Data are mean $\pm \operatorname{SEM}(n=6$ rats per group). $\mathrm{F}=6.58, \mathrm{df}=2$. ${ }^{\star} P<0.05$ versus TAT treatment; ${ }^{\#} P<0.05$ versus rats fed a low-salt diet. In the absence of ZO-1 antibodies in the immunoprecipitation, no ZO-1 was detected by Western blot analysis (not shown).

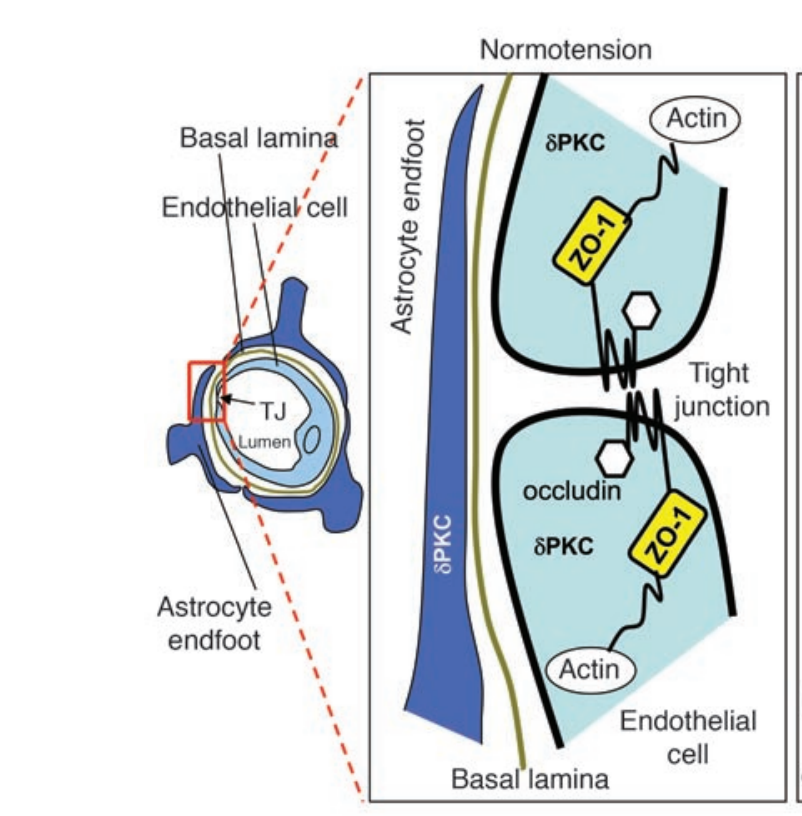

might have been responsible for the amelioration of neurological deficits and the subsequent increase in survival in the absence of an effect on blood pressure. Thus, the mechanism is distinct from that of kallikrein, a protein implicated in the pathogenesis of hypertension and renal diseases, which was shown to decrease the mortality rate from stroke in the DS rat model by reducing hypertension (41).

In normal control animals, $\mathrm{PKC}$ inhibition (or activation) exerts no effect, including no effect on heart rate and blood pressure (data not shown). Moreover, $\delta V 1-1$ has been given to patients with acute myocardial infarction with no adverse effects (42). Under ischemia/reperfusion conditions, the benefits of $\delta P K C$ inhibition are numerous - it reduces apoptosis (43), necrosis (29,

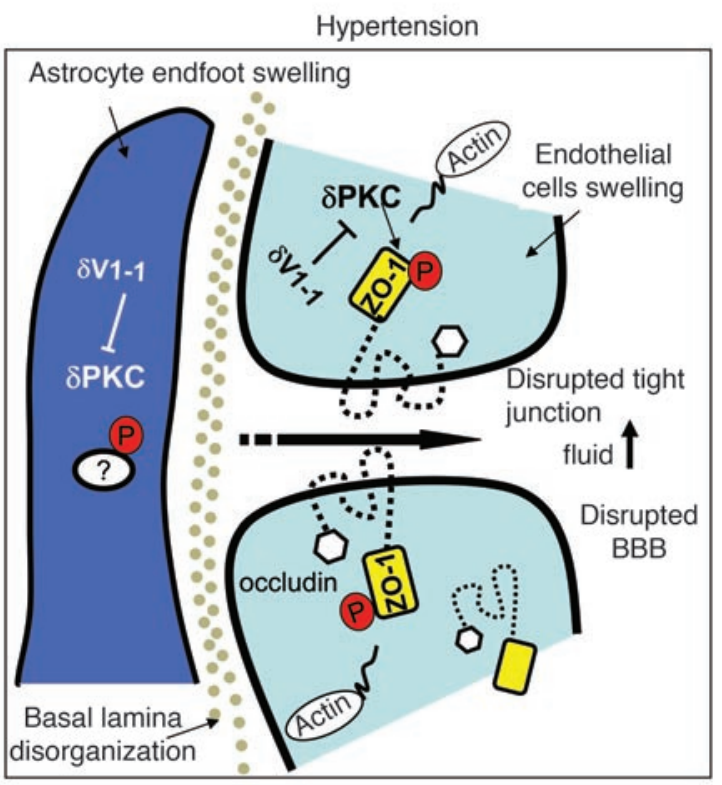

\section{Figure 8}

Scheme depicting a possible mechanism of $\delta P K C$-mediated tight junction dysfunction, increased BBB permeability leading to hypertensive encephalopathy, and potential sites of action of the $\delta P K C$ inhibitor $\delta V 1-1$. Left: scheme of a cerebral microvessel. Middle: enlargement of the area indicated in the red box at left. Right: scheme of a similar area in a hypertensive animal. Hypertension causes swelling of endothelia and endfeet of astrocytes surrounding the small vessels of brain and increases immunoreactivity of $\delta$ PKC (right versus middle). Serine and threonine phosphorylation of ZO-1 by $\delta$ PKC activation leads to the disruption of ZO-1 in the microvasculature and reduces its level in the tight junction (TJ). Consequently, BBB permeability increased. SPKC accumulation in endfeet is likely to lead to increased phosphorylation, but the substrates have not yet been identified. Inhibition of $\delta P K C$ by $\delta V 1-1$ inhibits ZO-1 phosphorylation, which, in turn, reduces changes in TJ morphology of the microvasculature and increases BBB impermeability. 
44), and infarct size in the heart (29) and the brain (24). SPKC inhibition also inhibits pyruvate dehydrogenase kinase activity and thus accelerates ATP regeneration (45). Furthermore, we previously showed that the protective effects of acute treatment with $\delta V 1-1$ in a transient cerebral ischemia model were mediated, at least in part, by the inhibition of apoptosis (24). We also found that the increased blood flow induced by $\delta V 1-1$ treatment in the ischemic model may play a role in neuroprotection (46). However, the mechanisms underlying hypertension-induced cerebral damage in DS rats are not identical to those of the transient cerebral ischemia model, and $\delta V 1-1$ may exert beneficial effects in DS rats via a different mechanism, the nature of which remains to be fully elucidated.

A number of studies have shown that activation of PKC regulates increased vascular permeability in many cell culture models. However, conflicting data on the critical role of $\mathrm{PKC}$ in this process have been described. Hypoxia and posthypoxic reoxygenation-induced increases in BBB paracellular permeability activate several $\mathrm{PKC}$ isozymes in rat brain microvessel endothelial cells (47). Overexpression of $\beta$ IPKC in human dermal microvascular endothelial cells augments phorbol ester-stimulated increases in albumin permeability (48), whereas overexpression of a dominant negative $\alpha \mathrm{PKC}$ in these cells interferes with the bryostatin-mediated decrease in transepithelial electrical resistance - a marker of barrier function (49). Furthermore, overexpression of $\delta$ PKC decreases transepithelial electrical resistance in LLC-PK1 cells, which are derived from pig kidney cortex (50). Oxidants induce loss of intestinal epithelial barrier integrity through the activation of $\delta$ PKC (51), and $\delta P K C$ is required for PMA- and 1,2-diacylglycerol-induced hyperpermeability in pulmonary microvascular endothelial cells (52). Thus, whereas these cell culture studies have shown the potential role of $\alpha, \beta \mathrm{I}$, and $\delta \mathrm{PKC}$ in barrier permeability, there is still some uncertainty about the specific roles of the isozymes. Our study is the first to our knowledge to provide direct evidence of BBB disruption caused by $\delta$ PKC activation in an in vivo animal model. Although $\beta$ IPKC is activated in the DS rat model (30), it is unlikely to be critical in this function because the $\beta$ IPKC inhibitor did not affect the incidence of encephalopathy (data not shown).

We also elucidated a potential molecular basis for the $\delta \mathrm{PKC}$ neuron-protective effect as depicted schematically in Figure 8. Inhibition of $\delta$ PKC in endothelial cells and capillary barrier-associated astrocyte endfeet in DS rat brain decreased endothelial and astrocyte swelling and BBB breakdown (Figure 8). Treatment with $\delta V 1-1$ significantly blocked the change in subcellular location of ZO-1 and occludin at the tight junction, which suggests that the modification of tight junction proteins by $\delta \mathrm{PKC}$ is responsible for the increase in BBB permeability caused by hypertension (Figure 8). Furthermore, we found that $\mathrm{ZO}-1$ was phosphorylated at serine and threonine residues and that $\delta V 1-1$ treatment inhibited the phosphorylation of $\mathrm{ZO}-1$. Indeed, serine/threonine phosphorylation changes are known to regulate the function of both existing and assembling tight junction proteins (53), and increased serine and threonine phosphorylation of $\mathrm{ZO}-1$ enhances $\mathrm{BBB}$ permeability $(36,40,54)$. Because a lack of $Z \mathrm{O}-1$ prevents the targeting of occludin to the junctional contacts, changes in $\mathrm{ZO}-1$ localization at the tight junction are coupled to alterations in the recruitment of other tight junction proteins at endothelial membranes and consequently lead to an increase in vascular permeability $(8,10)$. Thus, $\mathrm{ZO}-1$ may serve as a direct target for $\mathrm{SPC}$ in hypertensive encephalopathy or may be a substrate of a downstream kinase of $\delta \mathrm{PKC}$.
Alterations in the $\mathrm{BBB}$ and tight junctions correlate with reduced AQP immunoreactivity in perivascular glial endfeet (34, 35). This finding supports our data, which indicate that swollen and/or degenerated glial endfeet with a reduction in AQP4 content is relevant to alterations in tight junction proteins. AQP4 can be phosphorylated by PKC in vitro (54), and activation of PKC by phorbol 12,13-dibutyrate decreases AQP4 levels in astrocytes via phosphorylation at Ser180 (55). In the present study, we found that hypertension induced a significant increase in $\delta P K C$ levels and a decrease in AQP4 in the surrounding vessel wall. Furthermore, both $\delta \mathrm{PKC}$ and AQP4 were normalized by treatment with the $\delta P K C$ inhibitor $\delta V 1-1$. It is possible that activation of $\delta P K C$ in astrocytes induced by hypertension decreases AQP4 levels via phosphorylation, which, in turn, leads to astrocyte swelling and a subsequent increase in BBB permeability. Activated astrocytes can release inflammatory mediators such as tumor necrosis factor and interleukin-1, which can also increase the permeability of the BBB $(56,57)$. Moreover, it was shown in SPKC-null mice that neutrophil infiltration into the brain is reduced and proinflammatory cytokines and chemokines are inhibited following stroke (25). Thus, it is possible that increased $\delta \mathrm{PKC}$ in astrocytes following hypertension may, in part, be due to astrocyte-derived inflammatory factors. However, this remains to be determined.

In the present study, we also examined the immunoreactivity of $\delta P K C$ in postmortem samples of human brain to determine whether the same alteration of $\delta$ PKC activation in the microvasculature occurs in humans suffering from hypertension (see Supplemental Methods; supplemental material available online with this article; doi:10.1172/JCI32636DS1). Data for 3 male patients are provided in Supplemental Figure 1. Patients 07A22 and 07A29 both had a history of hypertension, whereas the third patient (07A31) had no history of hypertension and died of amyotrophic lateral sclerosis (normal basal ganglia and cortex were observed). All the samples show staining of the vessel wall smooth muscle. However, it appears that the 2 patients with a history of hypertension have more $\delta$ PKC staining in the arteriolar endothelial cells and astrocytes than does the nonhypertensive patient (Supplemental Figure 1).

Obviously, the number of samples in this study was too small to be conclusive, but these data are consistent with our animal data and suggest that hypertension-induced pathology in human brains may be associated with SPKC activation in the cerebral microvasculature. A larger study with many more samples, including age- and sex-matched patients with similar comorbidity factors, such as diabetes, obesity, and smoking habits, is required to make a definitive conclusion.

In conclusion, our findings suggest that $\delta \mathrm{PKC}$ activation is a key signaling event that dysregulates the structural and functional integrity of tight junctions and BBB, which leads to brain damage and hypertension-induced encephalopathy. Our data suggest that $\delta V 1-1$ has therapeutic potential for the prevention or reduction of cerebrovascular injury in hypertension-induced encephalopathy in rats. If a similar mechanism occurs in humans, a $\delta$ PKC inhibitor may be useful for patients at risk of hypertension-induced encephalopathy and stroke, common causes of morbidity and mortality, especially among people of African and Asian descent.

\section{Methods}

Protease and phosphatase inhibitor cocktails, trichloroacetic acid, paraformaldehyde, and Evans blue dye were purchased from Sigma- 
Aldrich. Antibodies against $\delta \mathrm{PKC}$ were purchased from Santa Cruz Biotechnology Inc., antibodies against ZO-1 and occludin were purchased from Zymed Biotechnology, and GAPDH antibody, clone 6C5, was purchased from Advanced Immunochemical. Antibodies for the anti-serine 643 phosphorylation of $\delta \mathrm{PKC}$ and anti-serine and threonine phosphorylation were from Cell Signaling Technology. Anti-mouse IgG and anti-rabbit IgG, peroxidase-linked species-specific antibodies, were purchased from Amersham Biosciences. Anti-CD31 and anti-AQP4 were from Chemicon. The $\delta$ PKC-specific antagonist peptide $\delta V 1-1$ ( $\delta$ PKC inhibitor, amino acids 8-17 [SFNSYELGSL]) was synthesized by American Peptides and conjugated to TAT-carrier peptide (amino acids 47-57) via a cysteine-cysteine $\mathrm{S}-\mathrm{S}$ bond at their $\mathrm{N}$ termini, as previously described (29).

Animals and treatments. Male DS rats, aged 4-5 weeks, were obtained from Harlan. The rats were fed a high-salt diet containing $8 \% \mathrm{NaCl}$ from the age of 6 weeks and were maintained to 15 weeks of age. From the age of 11 weeks, the rats were subcutaneously infused with $0.9 \%$ saline, control carrier TAT, or $\delta \mathrm{V} 1-1(1.0 \mathrm{mg} / \mathrm{kg} / \mathrm{d})$ with an osmotic pump (Alzet Osmotic Pump). The rats were monitored twice daily for behavioral signs of hypertensive encephalopathy. Major neurological findings and symptoms included the occurrence of seizures, head and forelimb repetitive twitching behaviors, fore- and hind-limb paralysis, and severe lethargy. If one of these symptoms occurred, they were regarded as a sign of hypertensive encephalopathy. The systolic blood pressure was measured biweekly with a tail-pulse pick-up method without anesthesia. Fractional shortening was measured by transthoracic echocardiography (Vivid 7; GE). The animals were euthanized at the end of 15 weeks. The animals were coded so that the observer was blinded to the treatment.

Tissue preparation. The animals were deeply anesthetized with isofluorene (3\%) and transcardially perfused with PBS. The brains of some animals were removed immediately and quickly frozen in liquid nitrogen. Some animals were transcardially perfusion-fixed with $4 \%$ paraformaldehyde fixative and further postfixed in paraformaldehyde for $1 \mathrm{~h}$. The brains were then cryoprotected by immersion in a $30 \%$ sucrose solution for $24 \mathrm{~h}$ at $4^{\circ} \mathrm{C}$ and then frozen rapidly in Tissue-Tek (Fisher Biotechnology) for immunostaining. All samples were stored at $-80^{\circ} \mathrm{C}$.

Measurement of BBB permeability. Rats were deeply anesthetized with isofluorene (3\%), and Evans blue dye ( $30 \mathrm{mg} / \mathrm{ml}$ in $0.9 \%$ saline) was injected (30 mg dye $/ \mathrm{kg}$ ) into the femoral vein. After 30 minutes, the rats were perfused with $200 \mathrm{ml}$ of PBS by transcardiac infusion, and the brains were removed. After the cerebellum and brainstem were removed, the cerebral hemispheres were homogenized in $2 \mathrm{ml}$ of $0.1 \mathrm{M}$ PBS. After centrifugation at $1,000 \mathrm{~g}$ for $5 \mathrm{~min}$ at $4^{\circ} \mathrm{C}, 0.7 \mathrm{ml}$ of the supernatant was added to $0.7 \mathrm{ml}$ of $100 \%(\mathrm{w} / \mathrm{v})$ trichloroacetic acid. The mixture was incubated at $4^{\circ} \mathrm{C}$ for $18 \mathrm{~h}$ and then centrifuged at $1,000 \mathrm{~g}$ for $30 \mathrm{~min}$ at $4^{\circ} \mathrm{C}$. Evans blue concentration in the supernatant was measured at $610 \mathrm{~nm}$ with a spectrophotometer. Results are presented as milligrams of Evans blue by comparing it with a standard solution.

Immunogold electron microscopy. Fixed specimens were rinsed with $10 \mathrm{mM}$ ammonium chloride in $0.1 \mathrm{M}$ PBS for $45 \mathrm{~min}$, dehydrated in an ascending ethanol series up to $70 \%$ ethanol, and embedded in the acrylic resin LR-Gold. The resin was hardened at $25^{\circ} \mathrm{C}$ under a 500 -W halogen lamp.

Thin sections were cut with an LKB V ultramicrotome and collected on formvar-coated nickel grids. The grids were incubated in $1 \%$ normal goat serum to block nonspecific reactions for $1 \mathrm{~h}$. The sections were incubated at room temperature with $\delta$ PKC antibodies (1:25) for $1 \mathrm{~h}$, washed with PBS, and incubated for $1 \mathrm{~h}$ with secondary antibodies (goat anti-rabbit for $\delta$ PKC, 1:50) coupled to gold particles. After a PBS wash, followed by a $1 \%$ lead citrate wash, the samples were examined with a Zeiss EM electron microscope.
Tissue fractionation. Frozen brain tissues were thawed on ice in homogenization buffer (20 mM Tris-HCl [pH 7.5], 2 mM EDTA, 10 mM EGTA, 250 $\mathrm{mM}$ sucrose, and protease and phosphatase inhibitors). The tissues were then minced and ground with a pestle in the presence of molecular grinding resin (Geno Technology) to produce homogenates. The homogenates were centrifuged at $100,000 \mathrm{~g}$ for $30 \mathrm{~min}\left(4^{\circ} \mathrm{C}\right)$ to separate the cytosolic soluble fraction from the membranous fraction. After the soluble fractions were isolated, the pellets were rehomogenized in homogenization buffer containing $1 \%$ Triton $\mathrm{X}-100$, incubated on ice for $30 \mathrm{~min}$, and subsequently centrifuged at $100,000 \mathrm{~g}$ for $30 \mathrm{~min}\left(4^{\circ} \mathrm{C}\right)$. The Triton-soluble particulate fraction was isolated, and the Triton-insoluble fraction pellets were dissolved in SDS sample buffer. The samples were analyzed by Western blot.

Western blot. Protein concentrations were determined by Bradford assay. Proteins from each fraction were resuspended in Laemmli buffer, loaded on $10 \%$ SDS-PAGE gels, and transferred onto nitrocellulose membranes. Membranes were probed with the indicated antibody followed by visualization by ECL and quantitated using NIH ImageJ software.

Immunofluorescence. Frozen 10- $\mu$ m-thick cryosections were used. The sections were allowed to thaw at room temperature for at least $2 \mathrm{~h}$ and then fixed in cold acetone for $2 \mathrm{~min}$. Primary anti-ZO-1 (1:50), anti-CD31 (1:500), or anti-occludin (1:10) antibodies were used. After 35 -min washings, sections were incubated with a mixture of FITC goat anti-rabbit antibody (1:50) and streptavidin (1:500) for $1 \mathrm{~h}$ in $1 \%$ blocking buffer. After incubation for $15 \mathrm{~min}$ in Hoechst stain, the sections were washed and examined under the microscope.

Immunohistochemistry. The indirect streptavidin-biotin-peroxidase method was used, and sections were treated with $0.3 \% \mathrm{H}_{2} \mathrm{O}_{2}$ for 5 minutes at room temperature. Sections were incubated in primary anti-rabbit AQP4 (1:200) antibodies for $2 \mathrm{~h}$ at room temperature. After the immunohistochemical reaction, sections were stained with an HRP IHC kit (Chemicon).

ZO-1 immunoprecipitation. Membrane fractions of brain homogenates (500 $\mu \mathrm{g}$ protein) were incubated with ZO-1 antibodies for $3 \mathrm{~h}$ at $4{ }^{\circ} \mathrm{C}$ and then incubated with Sepharose beads for $1 \mathrm{~h}$ at $4{ }^{\circ} \mathrm{C}$. The immunoprecipitates were separated on a $6 \%$ SDS-PAGE and transferred onto PVDF membranes. Serine and threonine phosphorylation of ZO-1 was determined by using a mixture of phosphoserine and phosphothreonine antibodies (1:1,000 dilution).

Statistics. All data are expressed as the mean \pm SEM. One-way ANOVA with a post-hoc Tukey's honestly significant difference test was used to determine difference between the groups. The F value with degrees of freedom (df) was provided. Survival was analyzed by conducting a standard Kaplan-Meier analysis with a log-rank test. The significance of changes in neurological symptoms was analyzed with a Fisher's exact test. A $P$ value less than 0.05 was considered significant.

\section{Acknowledgments}

This work was supported by NIH NS grant 044350 to Daria Mochly-Rosen.

Received for publication May 9, 2007, and accepted in revised form October 24, 2007.

Address correspondence to: Daria Mochly-Rosen, Department of Chemical and Systems Biology, Stanford University School of Medicine, CCSR, Room 3145A, 269 Campus Drive, Stanford, California 94305, USA. Phone: (650) 725-7720; Fax: (650) 723-4686; E-mail: mochly@stanford.edu.

Koichi Inagaki's present address is: Otsu Red-Cross Hospital, Shiga, Japan. 
1. Strandgaard, S., and Paulson, O.B. 1995. Cerebrovascular damage in hypertension. J. Cardiovasc. Risk. 2:34-39.

2. Phillips, S.J., and Whisnant, J.P. 1992. Hypertension and the brain. The National High Blood Pressure Education Program. Arch. Intern. Med. 152:938-945.

3. Baumbach, G.L., and Heistad, D.D. 1988. Cerebral circulation in chronic arterial hypertension. Hypertension. 12:89-95.

4. Conen, D., Bertel, O., and Dubach, U.C. 1987. Cerebral blood flow and calcium antagonists in hypertension. J. Hypertens. Suppl. 5:S75-S80.

5. Abbott, N.J., Ronnback, L., and Hansson, E. 2006. Astrocyte-endothelial interactions at the bloodbrain barrier. Nat. Rev. Neurosci. 7:41-53.

6. Hawkins, B.T., and Davis, T.P. 2005. The bloodbrain barrier/neurovascular unit in health and disease. Pharmacol. Rev. 57:173-185.

7. Ballabh, P., Braun, A., and Nedergaard, M. 2004. The blood-brain barrier: an overview: structure, regulation, and clinical implications. Neurobiol. Dis. 16:1-13.

8. Harhaj, N.S., and Antonetti, D.A. 2004. Regulation of tight junctions and loss of barrier function in pathophysiology. Int. J. Biochem. Cell. Biol. 36:1206-1237.

9. Huber, J.D., Egleton, R.D., and Davis, T.P. 2001. Molecular physiology and pathophysiology of tight junctions in the blood-brain barrier. Trends Neurosci. 24:719-725.

10. Wolburg, H., and Lippoldt, A. 2002. Tight junctions of the blood-brain barrier: development, composition and regulation. Vascul. Pharmacol. 38:323-337.

11. Davies, D.C. 2002. Blood-brain barrier breakdown in septic encephalopathy and brain tumours. J. Anat. 200:639-646.

12. Kalaria, R.N. 1992. The blood-brain barrier and cerebral microcirculation in Alzheimer disease. Cerebrovasc. Brain Metab. Rev. 4:226-260.

13. Nico, B., Roncali, L., Mangieri, D., and Ribatti, D. 2005. Blood-brain barrier alterations in MDX mouse, an animal model of the Duchenne muscular dystrophy. Curr. Neurovasc. Res. 2:47-54.

14. Prat, A., and Antel, J. 2005. Pathogenesis of multiple sclerosis. Curr. Opin. Neurol. 18:225-230.

15. Gasche, Y., and Copin, J.C. 2003. Blood-brain barrier pathophysiology and ischaemic brain oedema [In French]. Ann. Fr. Anesth. Reanim. 22:312-319.

16. Tanaka, C., and Nishizuka, Y. 1994. The protein kinase C family for neuronal signaling. Annu. Rev. Neurosci. 17:551-567.

17. Wolf, B.A., Easom, R.A., McDaniel, M.L., and Turk, J. 1990. Diacylglycerol synthesis de novo from glucose by pancreatic islets isolated from rats and humans. J. Clin. Invest. 85:482-490.

18. Johnson, A., Phillips, P., Hocking, D., Tsan, M.F., and Ferro, T. 1989. Protein kinase inhibitor prevents pulmonary edema in response to $\mathrm{H}_{2} \mathrm{O}_{2}$. Am J. Physiol. 256:H1012-H1022.

19. Ramirez, M.M., Kim, D.D., and Duran, W.N. 1996. Protein kinase $\mathrm{C}$ modulates microvascular permeability through nitric oxide synthase. Am. J. Physiol. 271:H1702-H1705.

20. Schaffler, A., Arndt, H., Scholmerich, J., and Palitzsch, K.D. 2000. Amelioration of hyperglycemic and hyperosmotic induced vascular dysfunction by in vivo inhibition of protein kinase $\mathrm{C}$ and p38 MAP kinase pathway in the rat mesenteric microcirculation. Eur. J. Clin. Invest. 30:586-593.

21. Meier, M., and King, G.L. 2000. Protein kinase C activation and its pharmacological inhibition in vascular disease. Vasc. Med. 5:173-185.

22. Hempel, A., et al 1999. Calcium antagonists ameliorate ischemia-induced endothelial cell permeability by inhibiting protein kinase C. Circulation.
99:2523-2529.

23. Aiello, L.P., et al. 1997. Vascular endothelial growth factor-induced retinal permeability is mediated by protein kinase $\mathrm{C}$ in vivo and suppressed by an orally effective beta-isoform-selective inhibitor. Diabetes. 46:1473-1480.

24. Bright, R., et al. 2004. Protein kinase C delta mediates cerebral reperfusion injury in vivo. J. Neurosci. 24:6880-6888.

25. Chou, W.H., et al. 2004. Neutrophil protein kinase $\mathrm{C}$ delta as a mediator of stroke-reperfusion injury. I. Clin. Invest. 114:49-56.

26. Knudsen, K.D., et al. 1970. Effects of chronic excess salt ingestion. Inheritance of hypertension in the rat. J. Exp. Med. 132:976-1000.

27. Smeda, J.S., and Payne, G.W. 2003. Alterations in autoregulatory and myogenic function in the cerebrovasculature of Dahl salt-sensitive rats. Stroke. 34:1484-1490

28. von Lutterotti, N., et al. 1992. Angiotensin II receptor antagonist delays renal damage and stroke in salt-loaded Dahl salt-sensitive rats. J. Hypertens. 10:949-957.

29. Chen, L., et al. 2001. Opposing cardioprotective actions and parallel hypertrophic effects of delta PKC and epsilon PKC. Proc. Natl. Acad. Sci. U. S. A. 98:11114-11119.

30. Inagaki, K., et al. 2002. Tissue angiotensin II during progression or ventricular hypertrophy to heart failure in hypertensive rats; differential effects on PKC epsilon and PKC beta. J. Mol. Cell. Cardiol. 34:1377-1385

31. Kraft, A.S., and Anderson, W.B. 1983. Phorbol esters increase the amount of $\mathrm{Ca}^{+}$, phospholipid-dependent protein kinase associated with plasma membrane. Nature. 301:621-623.

32. Spatz, M., and Klatzo, I. 1976. Pathological aspects of brain transport phenomena. Adv. Exp. Med. Biol. 69:479-495.

33. Rubin, L.L., and Staddon, J.M. 1999. The cell biology of the blood-brain barrier. Annu. Rev. Neurosci. 22:11-28.

34. Nicchia, G.P., et al. 2004. The role of aquaporin-4 in the blood-brain barrier development and integrity: studies in animal and cell culture models. Neuroscience. 129:935-945.

35. Nico, B., et al. 2003. Severe alterations of endothelial and glial cells in the blood-brain barrier of dystrophic mdx mice. Glia. 42:235-251.

36. Chen, M.L., Pothoulakis, C., and LaMont, J.T. 2002. Protein kinase $\mathrm{C}$ signaling regulates $\mathrm{ZO}-1$ translocation and increased paracellular flux of T84 colonocytes exposed to Clostridium difficile toxin A J. Biol. Chem. 277:4247-4254.

37. Willott, E., et al. 1993. The tight junction protein ZO-1 is homologous to the Drosophila discs-large tumor suppressor protein of septate junctions. Proc. Natl. Acad. Sci. U. S. A. 90:7834-7838.

38. Antonetti, D.A., et al. 1999. Vascular endothelial growth factor induces rapid phosphorylation of tight junction proteins occludin and zonula occluden 1. A potential mechanism for vascular permeability in diabetic retinopathy and tumors. J. Biol. Chem. 274:23463-23467.

39. Fischer, S., Wobben, M., Marti, H.H., Renz, D. and Schaper, W. 2002. Hypoxia-induced hyperpermeability in brain microvessel endothelial cells involves VEGF-mediated changes in the expression of zonula occludens-1. Microvasc. Res. 63:70-80.

40. Ohtake, K., et al. 2003. Poly-L-arginine enhances paracellular permeability via serine/threonine phosphorylation of ZO-1 and tyrosine dephosphorylation of occludin in rabbit nasal epithelium. Pharm. Res. 20:1838-1845.

41. Zhang, J.J., Chao, L., and Chao, J. 1999. Adenovirus- mediated kallikrein gene delivery reduces aortic thickening and stroke-induced death rate in Dahl salt-sensitive rats. Stroke. 30:1925-1931; discussion 1931-1932.

42. Roe, M. 2007. Targeted inhibition of delta-protein kinase $\mathrm{C}$ to ameliorate reperfusion injury during primary percutaneous coronary intervention for acute ST-elevation myocardial infarction: results from the DELTA MI trial. In American College of Cardiology 56th Annual Scientific Session, i2 Summit 2007 (Innovation in Intervention) Late-Breaking Clinical Trials II, Session 2405. March 24-27. New Orleans, Louisiana, USA.

43. Murriel, C.L., Churchill, E.N., Inagaki, K., Szweda, L.I., and Mochly-Rosen, D. 2004. Protein kinase Cdelta activation induces apoptosis in response to cardiac ischemia and reperfusion damage: a mechanism involving BAD and the mitochondria. J. Biol. Chem. 279:47985-47991.

44. Inagaki, K., et al. 2003. Inhibition of delta-protein kinase $\mathrm{C}$ protects against reperfusion injury of the ischemic heart in vivo. Circulation. 108:2304-2307.

45. Churchill, E.N., Murriel, C.L., Chen, C.H., MochlyRosen, D., and Szweda, L.I. 2005. Reperfusioninduced translocation of deltaPKC to cardiac mitochondria prevents pyruvate dehydrogenase reactivation. Circ. Res. 97:78-85.

46. Bright, R., Steinberg, G.K., and Mochly-Rosen, D. 2007. DeltaPKC mediates microcerebrovascular dysfunction in acute ischemia and in chronic hypertensive stress in vivo. Brain Res. 1144:146-155.

47. Fleegal, M.A., Hom, S., Borg, L.K., and Davis, T.P. 2005. Activation of PKC modulates blood-brain barrier endothelial cell permeability changes induced by hypoxia and posthypoxic reoxygenation. Am.J. Physiol. Heart Circ. Physiol. 289:H2012-H2019.

48. Nagpala, P.G., Malik, A.B., Vuong, P.T., and Lum, H. 1996. Protein kinase $C$ beta 1 overexpression augments phorbol ester-induced increase in endothelial permeability. J. Cell. Physiol. 166:249-255.

49. Clarke, H., et al. 2000. The transient increase of tight junction permeability induced by bryostatin 1 correlates with rapid downregulation of protein kinase C-alpha. Exp. Cell Res. 261:239-249.

50. Mullin, J.M., et al. 1998. Overexpression of protein kinase C-delta increases tight junction permeability in LLC-PK1 epithelia. Am. J. Physiol. 275:C544-C554

51. Banan, A., et al. 2002. Activation of delta-isoform of protein kinase $\mathrm{C}$ is required for oxidant-induced disruption of both the microtubule cytoskeleton and permeability barrier of intestinal epithelia. J. Pharmacol. Exp. Ther. 303:17-28.

52. Tinsley, J.H., Teasdale, N.R., and Yuan, S.Y. 2004. Involvement of PKCdelta and PKD in pulmonary microvascular endothelial cell hyperpermeability. Am. J. Physiol. Cell Physiol. 286:C105-C111.

53. Clarke, H., Marano, C.W., Peralta Soler, A., and Mullin, J.M. 2000. Modification of tight junction function by protein kinase C isoforms. Adv. Drug Deliv. Rev. 41:283-301.

54. Han, Z., Wax, M.B., and Patil, R.V. 1998. Regulation of aquaporin-4 water channels by phorbol esterdependent protein phosphorylation. J. Biol. Chem. 273:6001-6004

55. Zelenina, M., Zelenin, S., Bondar, A.A., Brismar, H., and Aperia, A. 2002. Water permeability of aquaporin- 4 is decreased by protein kinase $\mathrm{C}$ and dopamine. Am. J. Physiol. Renal Physiol. 283:F309-F318.

56. Aschner, M. 1998. Immune and inflammatory responses in the CNS: modulation by astrocytes. Toxicol. Lett. 102-103:283-287.

57. Abbott, N.J. 2002. Astrocyte-endothelial interactions and blood-brain barrier permeability. J. Anat. 200:629-638. 